\title{
Analytical Method to Interpret Displacement in Elastic Anisotropic Soil due to a Tunnel Cavity with an Arbitrary Cross Section
}

\author{
Qiongfang Zhang $\mathbb{D}^{1,2}$ Kang Cheng $\mathbb{D}^{1,2,4}$ Yadong Lou ${ }^{\mathbb{D}},{ }^{5}$ Tangdai Xia, ${ }^{4}$ Panpan Guo $\mathbb{D}^{4},{ }^{4}$ \\ and Xiaolu Gan iD 4 \\ ${ }^{1}$ Power China Huadong Engineering Corporation, Zhejiang, Hangzhou 310014, China \\ ${ }^{2}$ Engineering Research Center of Smart Rail Transportation of Zhejiang Province, Zhejiang, Hangzhou 310014, China \\ ${ }^{3}$ China Railway 11th Bureau Group Co., Ltd., Wuhan 430061, China \\ ${ }^{4}$ Research Center of Coastal and Urban Geotechnical Engineering, Zhejiang University, Hangzhou 310058, China \\ ${ }^{5}$ Hangzhou Metro Group Co., Ltd., Zhejiang, Hangzhou 310003, China
}

Correspondence should be addressed to Yadong Lou; 21212107@zju.edu.cn

Received 4 March 2021; Revised 5 May 2021; Accepted 1 June 2021; Published 3 July 2021

Academic Editor: Xin-Jiang Wei

Copyright (c) 2021 Qiongfang Zhang et al. This is an open access article distributed under the Creative Commons Attribution License, which permits unrestricted use, distribution, and reproduction in any medium, provided the original work is properly cited.

\begin{abstract}
Based on complex variable theory and conformal mapping method, the paper presents full plane elastic solutions around an unlined tunnel with arbitrary cross section in anisotropic soil. The solutions describe soil elastic solutions for assuming that the displacement vectors along the tunnel boundary are directed towards the center of the tunnel. Tunnels with different cross sections are used to illustrate the method and its correctness. An elliptical unlined tunnel case is discussed in detail in the paper. Using the image method, an approximate solution for predicting surface displacement and subsurface horizontal displacement around an unlined tunnel in anisotropic soil can be obtained. The results show anisotropic stiffness properties $n\left(n=E_{h} / E_{v}\right)$ and $m(m=$ $G_{v h} / E_{v}$ ) have a great effect on the displacement distribution patterns around an elliptical tunnel with certain shape.
\end{abstract}

\section{Introduction}

Due to the complexity of metro projects, many metro shields use varying cross-sectional shapes for the shield, such as rectangular [1], quasi-rectangular [2, 3], elliptical, horseshoe-shaped, and double-O-tube [4]. Research on tunnels with arbitrary cross sections can guide engineers in predicting ground displacement and underground deformation.

Currently, numerous research efforts have focused on ground displacement caused by circular shield tunnels. In this context, four primary methods are employed-empirical formula, analytical, model test, and numerical methods. Although numerical methods can easily identify the elastic solution for an underground excavation, analytical methods provide important information.
Carranzatorres and Fairhurst [5] stated that closed-form results can help engineers assess the general accuracy of numerical analysis and provide valuable means for obtaining insights into the general nature of a solution. Elasticity problems can be solved by combining the complex variable method with numerous theorems arising from analytical functions, such as Cauchy's integral theorem, Laurent's theorem, and theorems on conformal mapping $[6,7]$. These theories are widely used for the first fundamental problem of deep tunnels. Bobet [8] developed analytical solutions for a lined deep tunnel with a circular cross section in transversely isotropic rock using the Lkehnitskii formalism [9]. Zhang and Sun [10] derived an analytical solution for the radial displacement of an unlined deep tunnel with an arbitrary cross section in transversely isotropic rock using Kolosov-Muskhelishvili complex potentials [11]. Exadaktylos 
[12] and Manh [13] proposed closed-form solutions for the stress and displacement around an unlined deep tunnel with an arbitrary cross section in elastic isotropic and anisotropic ground, respectively. Exadaktylos [12] applied the method of Kolosov-Muskhelishvili complex potentials, whereas Manh [13] used Green's theory [14-16].

Analytical solutions have been reported for displacement-prescribed problems (also known as the second fundamental problem) in the context of unlined shallow tunnels in elastic soil. Moreover, analytical solutions have been developed for ground movements induced by tunnels with a circular cross section in clay by using the complex variable method. Sagaseta [17] considered tunnel excavation as the radial convergence of a point to the tunnel axis in an elastic half plane. Verruijt [18] extended the work of Sagaseta for arbitrary Poisson ratios and proposed an elliptical deformation mode for the tunnel boundary. Sagaseta found that the deformation modes of the tunnel boundary significantly influence the distribution of stress and displacement at the surface and in the internal soil. Park $[19,20]$ proposed four deformation patterns for tunnel boundaries and established the analytical displacement of the soil due to deep and shallow buried tunnels in clay. Pinto [21] discussed vertical displacement of a circular tunnel undergoing uniform radial deformation or exhibiting an elliptical deformation mode. The elliptical deformation mode was verified by comparing the analytical results with the measured results. Zymnis [22, 23] extended the analytical solutions proposed by Pinto to account for the cross-anisotropic stiffness properties of the soil. These studies show that the ground displacement caused by excavation of a circular tunnel in clay can be accurately predicted if appropriate soil parameters and tunnel deformation patterns are considered.

Using finite element analysis, Simpson et al. [24] confirmed that anisotropy has significant effects on both the magnitude of the surface settlement and shape of the surface settlement trough, whereas nonlinear behavior has only subtle effects. Lee and Rowe [25] showed that the elastic cross-anisotropy of soil has a significant effect on the computed settlements above the tunnel.

Relatively few studies have focused on the displacementprescribed problem for a tunnel with an arbitrary cross section in anisotropic soil. In this paper, it is assumed that the tunnel is so long and the plane-strain condition is employed. Full-plane elastic solutions are proposed for an unlined tunnel with an arbitrary cross section subjected to certain deformation modes. Several typical tunnel shapes are used to illustrate the proposed method. To verify the accuracy of this method, results for an elliptical unlined tunnel in transversely isotropic soil with stiffness parameters that correspond to isotropic conditions are compared with that for isotropic soil. The elastic solutions derived for isotropic soil and London clay are also compared to analyze the effect of anisotropic stiffness. Moreover, analytical solutions of displacements in half plane are obtained by using a virtual image technique. The effect of stiffness parameters, $n$ and $m$, on the distribution of the predicted displacement for an unlined elliptical tunnel is also analyzed.

\section{General Equations}

2.1. General Equations for Anisotropic Soil. For plane-strain problems, the constitutive relation can be given as

$$
\left\{\begin{array}{c}
\varepsilon_{x} \\
\varepsilon_{y} \\
\gamma_{x y}
\end{array}\right\}=\left[\begin{array}{lll}
\beta_{11} & \beta_{12} & \beta_{16} \\
\beta_{12} & \beta_{22} & \beta_{26} \\
\beta_{16} & \beta_{26} & \beta_{66}
\end{array}\right]\left\{\begin{array}{c}
\sigma_{x} \\
\sigma_{y} \\
\tau_{x y}
\end{array}\right\},
$$

where the $\beta_{i j}$ coefficients can be expressed as material stiffness parameters $E_{1}, E_{2}, v_{1}, v_{2}, G_{2}$, and $\alpha_{0}$ by Zymnis [22], for example,

$$
\begin{aligned}
\beta_{11}= & \cos ^{2} \alpha_{0}\left(\frac{\cos ^{2} \alpha_{0}}{E_{1}}-\frac{v_{2} \sin ^{2} \alpha_{0}}{E_{2}}\right)+\sin ^{2} \alpha_{0}\left(\frac{\sin ^{2} \alpha_{0}}{E_{2}}-\frac{v_{2} \cos ^{2} \alpha_{0}}{E_{2}}\right) \\
& -E_{1}\left(\frac{v_{1} \cos ^{2} \alpha_{0}}{E_{1}}+\frac{v_{2} \sin ^{2} \alpha_{0}}{E_{2}}\right)+\frac{\sin ^{2} 2 \alpha_{0}}{4 G_{2}},
\end{aligned}
$$

where $\alpha_{0}$ is dip angle of plane with isotropic properties; $E_{1}$, $E_{2}$ are Young's moduli of the soil in a direction parallel and normal to the isotropic plane, respectively; $v_{1}$ is Poisson's ratio in the plane of isotropy; $v_{2}$ is Poisson's ratio in the $y^{\prime}$ direction due to strain in the $x^{\prime}$ direction; and $G_{v h}$ is the shear modulus for strain in the $y /$ direction.

For $\alpha_{0}=0$,

$$
\begin{aligned}
E_{1} & =E_{h}, \\
v_{1} & =v_{h h}, \\
E_{2} & =E_{v}, \\
v_{2} & =v_{v h}, \\
G_{2} & =G_{v h} .
\end{aligned}
$$

The following stiffness parameters are commonly used to measure the anisotropy of soil:

$$
\begin{gathered}
n=\frac{E_{h}}{E_{v}}, \\
m=\frac{G_{v h}}{E_{v}} .
\end{gathered}
$$

Chatzigiannelis and Whittle [21] conducted laboratory tests on elastic anisotropic parameters for various types of soil. Their results are summarized in Table 1.

The elastic parameters are further constrained by thermodynamic considerations [22], such as

$$
\begin{aligned}
& G_{v h}, E_{v}, E_{h}>0, \\
& 0<n<4, \\
&-1<n_{h h}<1, \\
& n_{h h}+2 n_{h v} n_{v h} \leq 1 .
\end{aligned}
$$

2.2. Compatibility Equation for Transverse Isotropy. The equation for equilibrium under plane-strain conditions is given as follows: 
TABLE 1: Transverse isotropic parameters of different types of soil as reported in the literature [21].

\begin{tabular}{lccccc}
\hline Soil & $E_{v}(\mathrm{MPa})$ & $n=E_{h} / E_{v}$ & $m=G_{v h} / E_{v}$ & $v_{v h}$ & $v_{h h}$ \\
\hline London clay & 112 & 2.11 & 0.64 & 0.25 & -0.19 \\
Gravel & 305 & 0.51 & 0.3 & 0.25 & 0.18 \\
Sand & 330 & 0.94 & 0.4 & 0.15 & 0.17 \\
Silts & 300 & 0.79 & 0.78 & 0.06 & 0.29 \\
Soft clays & 80 & 0.86 & 0.33 & 0.34 & 0.3 \\
Clays & 100 & 1.46 & 0.44 & 0.34 & 0.27 \\
Stiff clays & 110 & 1.23 & 0.46 & 0.28 & 0.13 \\
\hline
\end{tabular}

$$
\frac{\partial^{2} \varepsilon_{x}}{\partial y^{2}}+\frac{\partial^{2} \varepsilon_{y}}{\partial x^{2}}-\frac{\partial^{2} \gamma_{x y}}{\partial y \partial x}=0
$$

In the absence of body forces, the stresses can be derived from the Airy stress function $F$ as follows:

$$
\begin{gathered}
\sigma_{x}=\frac{\partial^{2} F}{\partial y^{2}}, \\
\sigma_{y}=\frac{\partial^{2} F}{\partial x^{2}}, \\
\tau_{x y}=-\frac{\partial^{2} F}{\partial x \partial y} .
\end{gathered}
$$
obtain

Substituting equations (1) and (6) into equation (5), we

$$
\beta_{22} \frac{\partial^{4} F}{\partial x^{4}}+\left(2 \beta_{12}+\beta_{66}\right) \frac{\partial^{4} F}{\partial x^{2} \partial y^{2}}+\beta_{11} \frac{\partial^{4} F}{\partial y^{4}}=0 .
$$

The general solution can be expressed in terms of two analytic functions $F_{1}, F_{2}$ and their conjugates:

$$
\begin{aligned}
F(x, y) & =2 \operatorname{Re}\left[F_{1}\left(z_{1}\right)+F_{2}\left(z_{2}\right)\right] \\
& =F_{1}\left(z_{1}\right)+\overline{F_{1}\left(z_{1}\right)}+F_{2}\left(z_{2}\right)+\overline{F_{2}\left(z_{2}\right)},
\end{aligned}
$$

where the overscript "-" denotes the complex conjugate and $z_{k}=x+\lambda_{k} y, k=1,2$ are arguments in the $z_{k}$ plane. This plane is obtained from the affine transformation as follows:

$$
\begin{aligned}
z_{k} & =x_{k}+i y_{k} \\
& =x+\lambda_{k} y,
\end{aligned}
$$

where $\lambda_{k}$ are the material eigenvalues.

By introducing equation (9) into equation (8), we can obtain the characteristic equation of $\lambda_{k}$ :

$$
\beta_{11} \lambda^{4}-2 \beta_{16} \lambda^{3}+\left(2 \beta_{12}+\beta_{66}\right) \lambda^{2}-2 \beta_{26} \lambda+\beta_{22}=0,
$$

where the roots of the equation are conjugate complex numbers, $\lambda_{k}, \overline{\lambda_{k}}, k=1,2$.

New generalized complex variable functions are introduced as follows:

$$
\begin{aligned}
\Phi_{k}\left(z_{k}\right) & =\frac{\mathrm{d} F_{k}}{\mathrm{~d} z_{k}}, \Phi_{k}^{\prime}\left(z_{k}\right) \\
& =\frac{\mathrm{d} \Phi_{k}}{\mathrm{~d} z_{k}},
\end{aligned}
$$

where $\Phi_{k}\left(z_{k}\right)$ can be assumed in the form of an infinite power series.

From equations (7) and (12), the stress components are related to the variable functions as follows:

$$
\begin{aligned}
\sigma_{x}(x, y) & =2 \operatorname{Re}\left[\lambda_{1}^{2} \Phi_{1}^{\prime}\left(z_{1}\right)+\lambda_{2}^{2} \Phi_{2}^{\prime}\left(z_{2}\right)\right], \\
\sigma_{y}(x, y) & =2 \operatorname{Re}\left[\Phi_{1}^{\prime}\left(z_{1}\right)+\Phi_{2}^{\prime}\left(z_{2}\right)\right], \\
\tau_{x y}(x, y) & =-2 \operatorname{Re}\left[\lambda_{1} \Phi_{1}^{\prime}\left(z_{1}\right)+\lambda_{2} \Phi_{2}^{\prime}\left(z_{2}\right)\right] .
\end{aligned}
$$

Additionally, the cavity is assumed to be free of forces along its surface. The displacement components are determined by integrating the strain components $\left(\varepsilon_{x}=\partial u / \partial x, \varepsilon_{y}=\partial v / \partial y, \gamma_{x y}=\partial u / \partial y+\partial v / \partial x\right)$ to obtain

$$
\begin{aligned}
& U(x, y)=2 \operatorname{Re}\left[p_{1} \Phi_{1}\left(z_{1}\right)+p_{2} \Phi_{2}\left(z_{2}\right)\right], \\
& V(x, y)=2 \operatorname{Re}\left[q_{1} \Phi_{1}\left(z_{1}\right)+q_{2} \Phi_{2}\left(z_{2}\right)\right],
\end{aligned}
$$

with

$$
\begin{aligned}
& p_{1}=\beta_{11} \lambda_{1}^{2}+\beta_{12}, \\
& p_{2}=\beta_{11} \lambda_{2}^{2}+\beta_{12}, \\
& q_{1}=\beta_{12} \lambda_{1}+\frac{\beta_{22}}{\lambda_{1}}, \\
& q_{1}=\beta_{12} \lambda_{2}+\frac{\beta_{22}}{\lambda_{2}} .
\end{aligned}
$$

\section{Closed-Form Solution Obtained by Conformal Mapping}

To find solutions for this problem, we first consider the transformation of tunnels with an arbitrary cross section, such as an ellipse, circle, or square, in the $z$ plane to a unit circular hole in the $\zeta$ plane, as shown in Figure 1. The transformation function can be assumed as

$$
\begin{aligned}
z & =w(\zeta) \\
& =R\left(\zeta+\sum_{n=1}^{N} a_{n} \zeta^{-n}\right), \quad \zeta=\rho e^{i \theta},
\end{aligned}
$$

where $R$ is a real number related to the size of the tunnel and $a_{n}$ are generally complex coefficients that satisfy $\left|a_{n}\right|<1 / n$ [26]. The inverse mapping function $w^{-1}(z)$ is analytic, single valued, and nonzero outside the tunnel boundary.

In many cases, it can be assumed that the physical domain possesses $p$ symmetry axes, which yields 


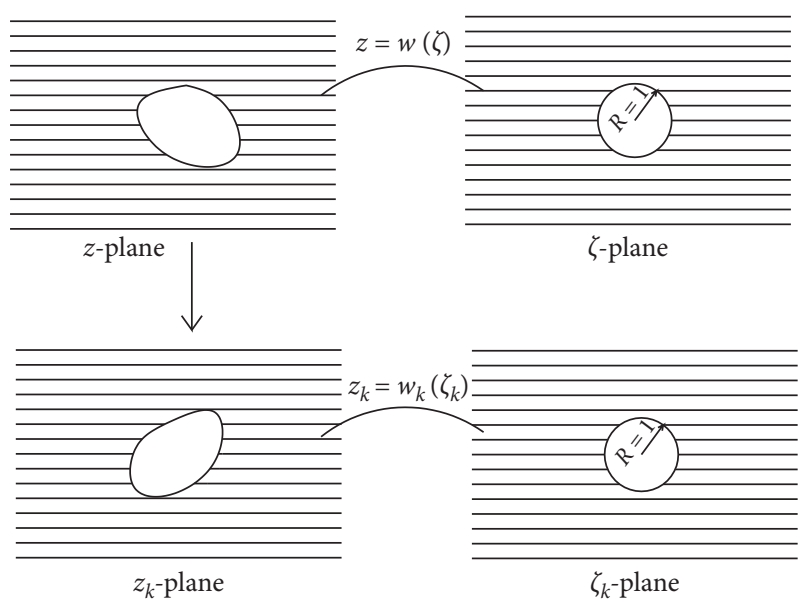

FIgURE 1: Conformal mapping of a region outside an unlined tunnel to the exterior region of a unit circle.

$$
\begin{aligned}
z & =w(\zeta) \\
& =R\left(\zeta+\sum_{n=1}^{N} a_{1-p n} \zeta^{1-p n}\right)
\end{aligned}
$$

Assuming that the central position of the tunnel boundary remains unchanged after deformation, the boundary remains axisymmetric along the $x$ and $y$ axes. After deformation, the curve can be expressed by

$$
\begin{aligned}
Z & =w_{c}(\zeta), \\
& =R_{c}\left(\zeta+\sum_{n=1}^{N} b_{n} \zeta^{-n}\right), \\
& =R\left(\frac{R_{c}}{R} \zeta+\frac{R_{c}}{R} \sum_{n=1}^{N} b_{n} \zeta^{-n}\right) .
\end{aligned}
$$

Because the soil is transversely isotropic, the boundary of the tunnel in the original plane ( $z$ plane) is affine transformed to cutouts in the $z_{k}$ planes:

$$
z_{k}=x_{k}+i y_{k}=x+\lambda_{k} y, \quad k=1,2 .
$$

The exterior region of an unlined tunnel in the $z_{k}$ plane can be mapped to the exterior region of a unit circle in the $\zeta_{k}$ plane. The conformal mapping functions corresponding to the original and deformed tunnel boundaries are as follows:

$$
\begin{aligned}
& z_{k}=w_{k}\left(\zeta_{k}\right)=R \sum_{n=1}^{N}\left(c_{k n} \zeta_{k}^{n}+d_{k n} \zeta_{k}^{-n}\right), \\
& Z_{k}=w_{k}\left(\zeta_{k}\right)=R \sum_{n=1}^{N}\left(g_{k n} \zeta_{k}^{n}+r_{k n} \zeta_{k}^{-n}\right), \quad\left|\zeta_{k}\right| \geq 1, \text { for } k=1,2,
\end{aligned}
$$

where the circles $\zeta_{k}=1$ correspond to the circle $\zeta=1$ and $\zeta_{k} \longrightarrow \infty$ when $\zeta \longrightarrow \infty$.
To ensure that the transformed equations (19) and (20) are single valued, all roots of $\mathrm{d} z_{k} / \mathrm{d} \zeta_{k}=0$ must be located inside the unit circle $\left|\zeta_{k}\right|=1$, thereby giving

$$
\left\{\begin{array}{l}
\left|\zeta_{k}^{2 n}\right|=\left|\frac{d_{k n}}{c_{k n}}\right|<1, \\
\left|\zeta_{k}^{2 n}\right|=\left|\frac{r_{k n}}{g_{k n}}\right|<1,
\end{array}\right.
$$

which should be located inside the unit circle. Thus, one-toone mapping is obtained.

Using equations (17) and (20), $z_{k}$ can also be expressed as

$$
\begin{aligned}
z_{k} & =\frac{z+\bar{z}}{2}+\lambda_{k} \frac{z-\bar{z}}{2 i} \\
& =\frac{R\left\{\left(i+\lambda_{k}\right)\left(\zeta+\sum_{n=1}^{N} a_{n} \zeta^{-n}\right)+\left(i-\lambda_{k}\right)\left(\zeta^{-1}+\sum_{n=1}^{N} \bar{a}_{n} \zeta^{n}\right)\right\}}{2 i} .
\end{aligned}
$$

By comparing the coefficients of equations (20) and (22) for the tunnel boundary $(\rho=1)$, one can obtain $c_{k n}$ and $d_{k n}$.

Hence,

$$
\left\{\begin{array}{l}
c_{k 1}=\frac{\left(1-\lambda_{k} i\right)+\left(1-\lambda_{k} i\right) \overline{a_{1}}}{2} \\
d_{k 1}=\frac{\left(1-\lambda_{k} i\right) a_{1}+\left(1-\lambda_{k} i\right)}{2} .
\end{array}\right.
$$

For $n \geq 2$,

$$
\left\{\begin{array}{l}
c_{k n}=\frac{\left(1-i \lambda_{k}\right) a_{n}}{2}, \\
d_{k n}=\frac{\left(1+i \lambda_{k}\right) \overline{a_{n}}}{2} .
\end{array}\right.
$$

Similarly,

$$
\left\{\begin{array}{l}
g_{k 1}=\frac{R_{c}}{R} \frac{\left(1-\lambda_{k} i\right)+\left(1+\lambda_{k} i\right) \overline{b_{1}}}{2} \\
r_{k 1}=\frac{R_{c}}{R} \frac{\left(1-\lambda_{k} i\right) b_{1}+\left(1+\lambda_{k} i\right)}{2}
\end{array}\right.
$$

For $n \geq 2$,

$$
\left\{\begin{array}{l}
g_{k n}=\frac{R_{c}}{R} \frac{\left(1-i \lambda_{k}\right) b_{n}}{2}, \\
r_{k n}=\frac{R_{c}}{R} \frac{\left(1+i \lambda_{k}\right) \overline{b_{n}}}{2} .
\end{array}\right.
$$

$\Phi_{k}\left(z_{k}\right)$ is assumed to have the following form:

$$
\Phi_{k}\left(z_{k}\right)=\sum_{n=1}^{N} A_{k n}\left(\zeta_{k}\right)^{-n} .
$$


The displacement components on the tunnel boundary can be obtained using equation (16):

$$
\begin{aligned}
& U(x, y)=2 \sum_{k=1}^{2}\left(p_{k} \sum_{n=1}^{N} A_{k n} e^{-i n \theta}+\overline{p_{k}} \sum_{n=1}^{N} \overline{A_{k n}} e^{i n \theta}\right), \\
& V(x, y)=2 \sum_{k=1}^{2}\left(q_{k} \sum_{n=1}^{N} A_{k n} e^{-i n \theta}+\overline{q_{k}} \sum_{n=1}^{N} \overline{A_{k n}} e^{i n \theta}\right) .
\end{aligned}
$$

It is assumed that the displacement vectors along the tunnel boundary are directed toward the center of the tunnel. The displacement $u_{k}$ at the tunnel boundary can be expressed as

$$
\begin{aligned}
u_{k}= & U(x, y)+\lambda_{k} V(x, y) \\
= & 2\left(p_{1}+\lambda_{k} q_{1}\right) \sum_{n=1}^{N} A_{1 n} e^{-i n \theta}+2\left(p_{2}+\lambda_{k} q_{2}\right) \sum_{n=1}^{N} A_{2 n} e^{-i n \theta} \\
& +2\left(\overline{p_{1}}+\lambda_{k} \overline{q_{1}}\right) \sum_{n=1}^{N} \overline{A_{1 n}} e^{-i n \theta}+2\left(\overline{p_{2}}+\lambda_{k} \overline{q_{2}}\right) \sum_{n=1}^{N} \overline{A_{2 n}} e^{-i n \theta}
\end{aligned}
$$

The total displacement $u_{k}$ at the tunnel boundary $\rho=1$ can also be expressed as

$$
\begin{aligned}
u_{k} & =Z_{k}-z_{k} \\
& =R \sum_{n=1}^{N}\left(\left(g_{k n}-c_{k n}\right) e^{i n \theta}+\left(r_{k n}-d_{k n}\right) e^{-i n \theta}\right) .
\end{aligned}
$$

Equating the coefficients for similar terms of $e^{i n \theta}$ and $e^{-i n \theta}$ in equations (29) and (30) yields

$$
\left\{\begin{array}{l}
2\left(p_{1}+\lambda_{k} q_{1}\right) A_{1 n}+2\left(p_{2}+\lambda_{k} q_{2}\right) A_{2 n}=R\left(r_{k n}-d_{k n}\right), \\
2\left(\overline{p_{1}}+\lambda_{k} \overline{q_{1}}\right) \overline{A_{1 n}}+2\left(\overline{p_{2}}+\lambda_{k} \overline{q_{2}}\right) \overline{A_{2 n}}=R\left(g_{k n}-c_{k n}\right) .
\end{array}\right.
$$

The above equations are satisfied by

$$
\left\{\begin{array}{l}
A_{1 n}=\frac{R\left(\left(r_{1 n}-d_{1 n}\right)\left(p_{2}+\lambda_{2} q_{2}\right)-\left(r_{2 n}-d_{2 n}\right)\left(p_{2}+\lambda_{1} q_{2}\right)\right)}{2\left(\lambda_{1}-\lambda_{2}\right)\left(p_{2} q_{1}-p_{1} q_{2}\right)} \\
A_{2 n}=\frac{R\left(\left(r_{1 n}-d_{1 n}\right)\left(p_{1}+\lambda_{2} q_{1}\right)-\left(r_{2 n}-d_{2 n}\right)\left(p_{1}+\lambda_{1} q_{1}\right)\right)}{2\left(\lambda_{1}-\lambda_{2}\right)\left(p_{1} q_{2}-p_{2} q_{1}\right)} \\
\overline{A_{1 n}}=\frac{R\left(\left(g_{1 n}-c_{1 n}\right)\left(\overline{p_{2}}+\lambda_{2} \overline{q_{2}}\right)-\left(g_{2 n}-c_{2 n}\right)\left(\overline{p_{2}}+\lambda_{1} \overline{q_{2}}\right)\right)}{2\left(\lambda_{1}-\lambda_{2}\right)\left(\overline{p_{2} q_{1}}-\overline{p_{1} q_{2}}\right)} \\
\overline{A_{2 n}}=\frac{R\left(\left(g_{1 n}-c_{1 n}\right)\left(\overline{p_{1}}+\lambda_{2} \overline{q_{1}}\right)-\left(g_{2 n}-c_{2 n}\right)\left(\overline{p_{1}}+\lambda_{1} \overline{q_{1}}\right)\right)}{2\left(\lambda_{1}-\lambda_{2}\right)\left(\overline{p_{1} q_{2}}-\overline{p_{2} q_{1}}\right)}
\end{array}\right.
$$

It can be seen that once the mapping function for the original and deformed tunnel boundaries and the parameters of elastic anisotropic soil are determined, the coefficients of equation (27) can be ascertained. The elastic solutions around the tunnel can be obtained using equations (12) and (13).

\section{Validation of the Proposed Solution with Known Solutions}

4.1. Circular Tunnel. For a cylindrical cavity of radius $R$ in infinite anisotropic soil, the boundary condition can be solved by mapping onto a circle of unit radius:

$$
z=R \zeta .
$$

The conformal mapping function of $z_{k}$ can be written as

$$
z_{k}=R\left(\zeta_{k}+\zeta_{k}^{-1}\right)
$$

When undergoing uniform convergence $u_{\varepsilon}$, the deformed tunnel boundary can be expressed as

$$
Z=\left(R-u_{\varepsilon}\right) \zeta
$$

The conformal mapping function of $z_{k}$ can be written as

$$
Z_{k}=\left(R-u_{\varepsilon}\right)\left(\zeta_{k}+\zeta_{k}^{-1}\right) \text {. }
$$

When subjected to the ovalization mode $u_{\delta}$, the deformed tunnel boundary can be expressed as

$$
Z=R\left(\zeta+\frac{u_{\delta}}{R} \zeta^{-1}\right)
$$

The conformal mapping function of $z_{k}$ can be written as 


$$
Z_{k}=R\left(\frac{\left(1-\lambda_{k} i\right)+\left(1+\lambda_{k} i\right)\left(u_{\delta} / R\right)}{2} \zeta_{k}+\frac{\left(1-\lambda_{k} i\right)\left(u_{\delta} / R\right)+\left(1+\lambda_{k} i\right)}{2} \zeta_{k}^{-1}\right)
$$

The expression for the displacement can be obtained using equations (13) and (32), which is identical to the solution obtained by Zymnis [22].

4.2. Nonelliptical Tunnels. It is useful to determine the coefficients of approximate polynomial mapping functions.
This problem has been previously addressed in literature. Heller [27] provided the mapping function for a rectangular opening of unit width and height $\mathrm{K}$, using the SchwarzChristoffel integral:

$$
z=R\left[\zeta+\frac{1-K^{2}}{4} \frac{1}{\zeta}-\frac{K^{2}}{24} \frac{1}{\zeta^{3}}-\frac{K^{2}\left(1-K^{2}\right)}{160} \frac{1}{\zeta^{5}}-\frac{K^{2}\left(1-3 K^{2}+K^{4}\right)}{896} \frac{1}{\zeta^{7}}+\ldots\right]
$$

For nonelliptical holes with rounded corners, we have the following approximate polynomial mapping functions [28]:

$$
\begin{aligned}
& z=R\left[\zeta+\frac{1}{3} \frac{1}{\zeta^{2}}+\frac{1}{45} \frac{1}{\zeta^{5}}\right] \text { (triangle), } \\
& z=R\left[\zeta-\frac{1}{6} \frac{1}{\zeta^{3}}+\frac{1}{56} \frac{1}{\zeta^{7}}\right] \text { (square), } \\
& z=R\left[\zeta+\frac{1}{2} \frac{1}{\zeta}-\frac{1}{8} \frac{1}{\zeta^{3}}-\frac{3}{80} \frac{1}{\zeta^{5}}\right]\left(\frac{\text { rectangle with length }}{\text { width ratio of 3.24: } 1}\right), \\
& z=R\left[\zeta+0.643 \frac{1}{\zeta}-0.098 \frac{1}{\zeta^{3}}-0.038 \frac{1}{\zeta^{5}}\right]\left(\frac{\text { rectangle with length }}{\text { width ratio of 5: } 1}\right)
\end{aligned}
$$

Manh [13] proposed that, to reduce the error for elastic fields at the corner of a rectangular opening, at least 10 terms must be used in the conformal mapping functions. Once an approximate polynomial mapping for the original and deformed shapes of the hole boundary is obtained, the elastic solution can be determined using the analytical method proposed herein.

\subsection{Elliptical Tunnel}

4.3.1. Solutions for an Unlined Elliptical Tunnel. Assuming that the ratio of the semi-major and semi-minor axes remains unchanged during uniform radial convergence of an elliptical tunnel boundary, as shown in Figure 2, we observe that

$$
\begin{aligned}
\frac{a_{1}}{a_{2}} & =\frac{b_{1}}{b_{2}} \\
& =\frac{\rho_{1}}{\rho_{2}} \\
& =\frac{1}{k},
\end{aligned}
$$

where $a_{1}, a_{2}$ are the semi-major axes of the elliptical tunnel before and after deformation, respectively; $b_{1}, b_{2}$ are the semi-minor axes of the elliptical tunnel before and after deformation, respectively; $\rho_{1}, \rho_{2}$ are the extreme diameter of any point on the elliptical tunnel before and after deformation, respectively; and $k$ is a length ratio.

For an elliptical cavity illustrated in Figure 2 in an infinite medium undergoing uniform convergence, $u_{x}$ and $u_{y}$, the displacement components at the tunnel wall, can be expressed by

$$
\left\{\begin{array}{l}
u_{x}=-(1-k) x \\
u_{y}=-(1-k) y
\end{array}, \quad(0<k \leq 1), z=w(\zeta)=R\left(\zeta+m \zeta^{-1}\right),\right.
$$

where $R=\left(a_{1}+b_{1}\right) / 2, m=\left(a_{1}-b_{1}\right) /\left(a_{1}+b_{1}\right)$. The mapping function transforms the elliptical cavity to a unit circle as follows:

$$
\begin{aligned}
Z & =w(\zeta) \\
& =k R\left(\zeta+m \zeta^{-1}\right)
\end{aligned}
$$

Thereafter, we introduce the following mapping functions, $z_{k}\left(\zeta_{k}\right)$ and $Z_{k}\left(\zeta_{k}\right)$ :

$$
\begin{aligned}
& z_{k}=R\left(\frac{\left(1-\lambda_{k} i\right)+\left(1+\lambda_{k} i\right) m}{2} \zeta_{k}+\frac{\left(1-\lambda_{k} i\right) m+\left(1+\lambda_{k} i\right)}{2} \zeta_{k}^{-1}\right), \\
& Z_{k}=R\left(k \frac{\left(1-\lambda_{k} i\right)+\left(1+\lambda_{k} i\right) m}{2} \zeta_{k}+k \frac{\left(1-\lambda_{k} i\right) m+\left(1+\lambda_{k} i\right)}{2} \zeta_{k}^{-1}\right) .
\end{aligned}
$$




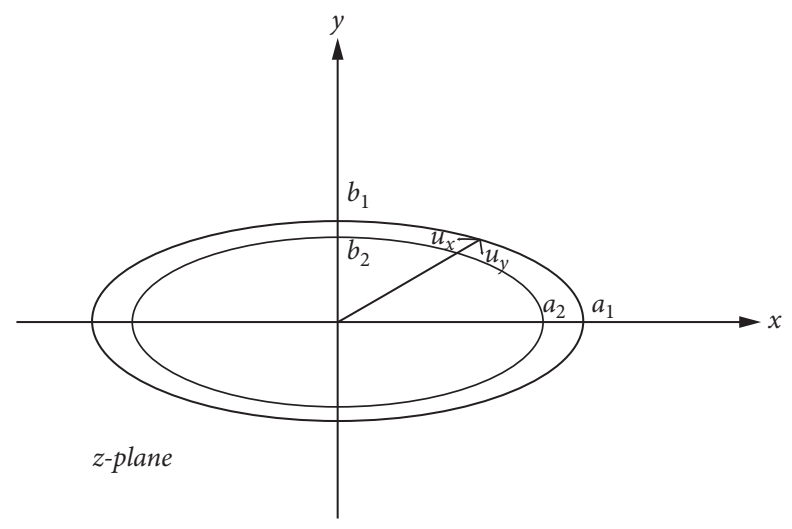

Figure 2: Original and transformed $(z)$ plane.

The coefficients $A_{1 n}$ and $A_{2 n}$ can be obtained from equation (32):

$$
\left\{\begin{array}{l}
A_{1 n}=\frac{(1-k)\left(q_{2} a_{1}-p_{2} i b_{1}\right)}{2\left(p_{2} q_{1}-p_{1} q_{2}\right)}, \\
A_{2 n}=\frac{(1-k)\left(-q_{1} a_{1}+p_{1} i b_{1}\right)}{2\left(p_{2} q_{1}-p_{1} q_{2}\right)}, \\
\overline{A_{1 n}}=\frac{(1-k)\left(\overline{q_{2}} a_{1}+\overline{p_{2}} i b_{1}\right)}{2\left(\overline{p_{2} q_{1}}-\overline{p_{1} q_{2}}\right)}, \\
\overline{A_{2 n}}=\frac{(1-k)\left(-\overline{q_{1}} a_{1}+\overline{p_{1}} b_{1} i\right)}{2\left(\overline{p_{1} q_{2}}-\overline{p_{2} q_{1}}\right)} .
\end{array}\right.
$$

When $a_{1}=b_{1}$, the results can be reduced to the results obtained by Zymnis [22].

4.3.2. Comparison with Isotropic Soil. In general, tunnel contours comprise complex curves; thus, to generalize these contours, it is reasonable to simplify complicated tunnels as unlined tunnels with an elliptical outline [29]. To verify the proposed method, we utilized approximately equal stiffness parameters in anisotropic soil; i.e., $n=E_{h} / E_{v}=1.0, v_{v h}$ $=v_{h h}=0.5, m=G_{v h} / E_{v}=0.335$. In addition, we compared the stress and displacement obtained with the proposed theoretical method with the results obtained by the method used in isotropic soil. Figures 3(a)-3(e) compare the distribution of elastic solutions around an elliptical tunnel with $a / H=0.1064 ; b / a=0.8$. The analyses can be applied under the assumption of incompressible behavior, when the soil is soft clay. The conditions for incompressibility have been given by Gibson [27] as $v_{v h}=0.5, v_{h h}=1-2 n v_{v h}^{2}=1-(n$ /2). Because the distribution of displacement does not depend on the magnitude of the elliptical tunnel and the distribution of stress does not depend on the magnitude of the modulus, the displacement and stress fields are given by the dimensionless coordinates, $x / H$ and $y / H$. The results for isotropic soil are shown for $x / H<0$, and the results for transversely isotropic soil are shown for $x / H>0$ in the following figures.
Figures 3(a)-3(e) indicate that when the boundary of an elliptical tunnel uniformly converges to the center of the tunnel, the absolute values of the results increase as we approach the boundary of the elliptical tunnel. Similarly, as we move farther away from the boundary of the elliptical tunnel, the absolute values of the results decrease more rapidly. In all the cases, the normal stress and horizontal displacement are symmetric with respect to the $x$ axis, whereas the shear stress and vertical displacement are antisymmetric with respect to the $y$ axis. Excellent agreement is observed between the results for isotropic and transversely isotropic soils, verifying that the anisotropic solutions obtained by the analytical method proposed herein can converge to the isotropic solution with appropriate stiffness parameters.

4.3.3. Comparison of Results for Isotropic Soil and London Clay. Gasparre [28] noted that London clay exhibits significant anisotropy at low strain levels, i.e., $<0.001 \%$. Figures 4(a)-4(e) compare the distribution of elastic solutions around an unlined elliptical tunnel with $b / a=0.8$, obtained for the isotropic model (with $\nu=0.5$ ) and London clay with stiffness parameters of $n=E_{h} / E_{v}=2.125$; $m=G_{v h} / E_{v}=1.125 ; v_{v h}=0.25 ; v_{h h}=-0.19$. The results for isotropic soil are shown for $x / H<0$, whereas the results for London clay are shown for $x / H>0$ in Figure 4.

Figures 4(a)-4(e) present a comparison of the analytical solutions for isotropic soil and London clay under uniform radial convergence deformation of an elliptical tunnel. The anisotropic parameters of London clay have little influence on the horizontal stress, horizontal displacement, and shear stress. However, a slightly faster attenuation of vertical stress and vertical displacement with distance is observed.

\section{Solutions of Ground Displacement in Half Plane}

5.1. Virtual Image Technique. The tunnel is embedded at a depth $H$ from the ground surface. In accordance with the study by Sagaseta, the ground movements associated with an unlined tunnel located at a depth $H$ below the traction-free ground surface can be approximately represented by a 


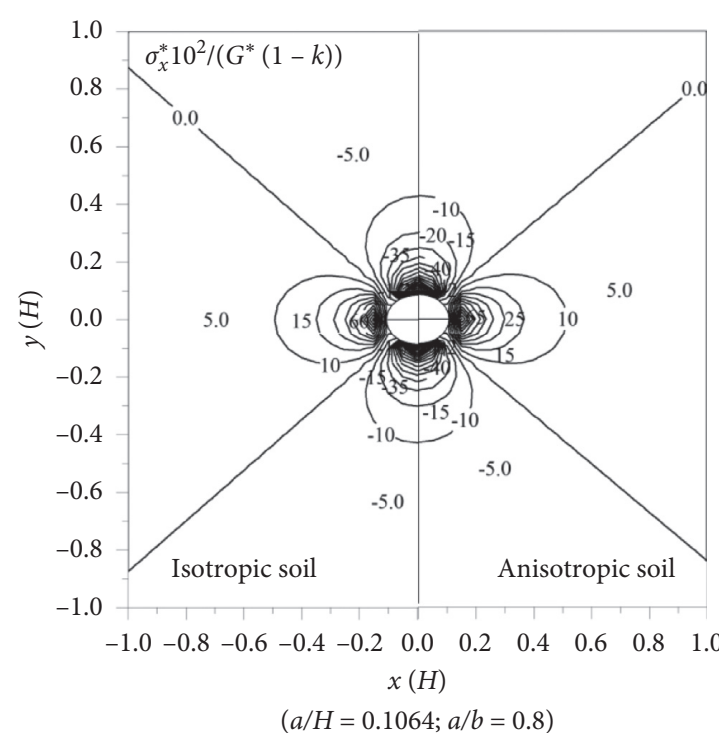

(a)

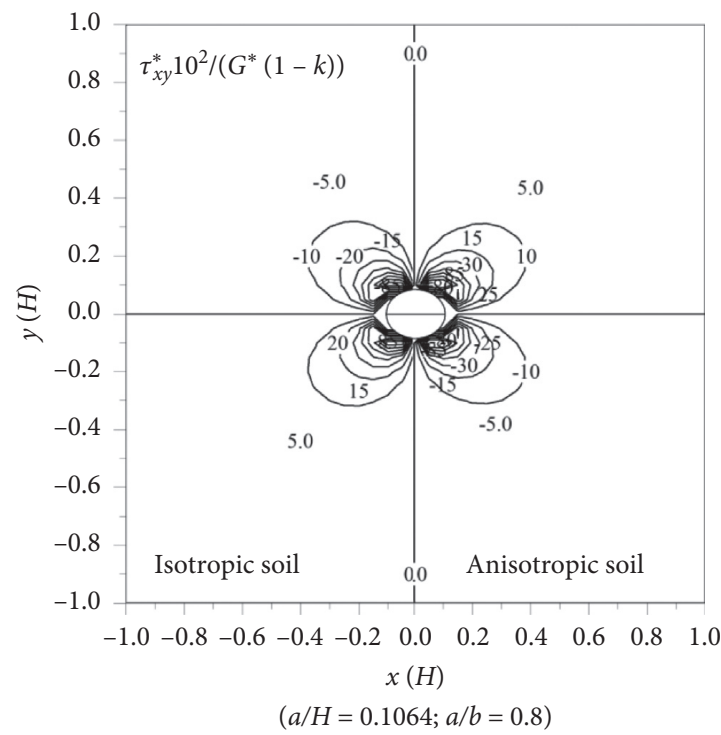

(c)

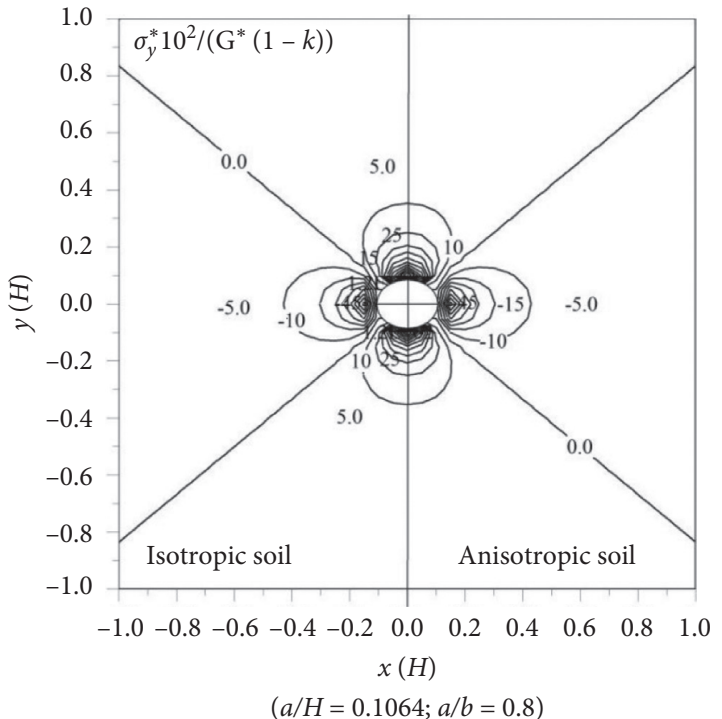

(b)

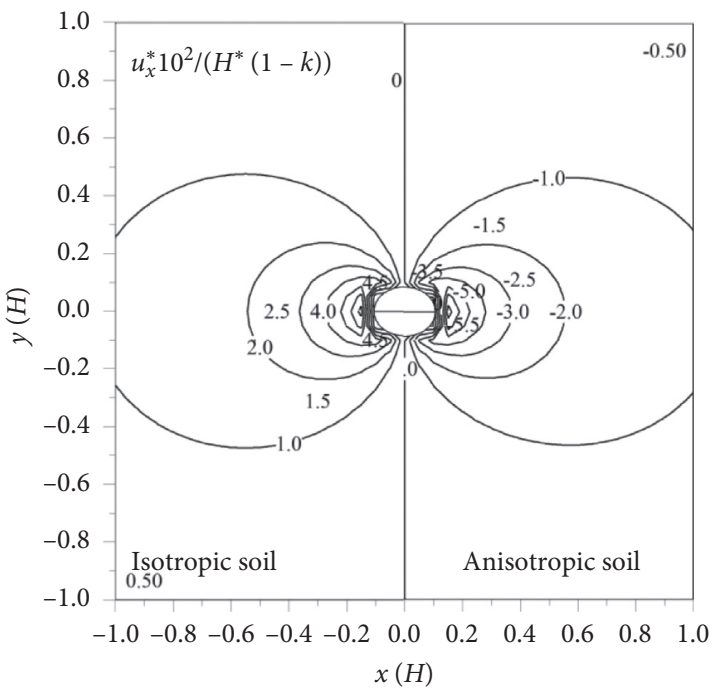

$(a / H=0.1064 ; a / b=0.8)$

(d)

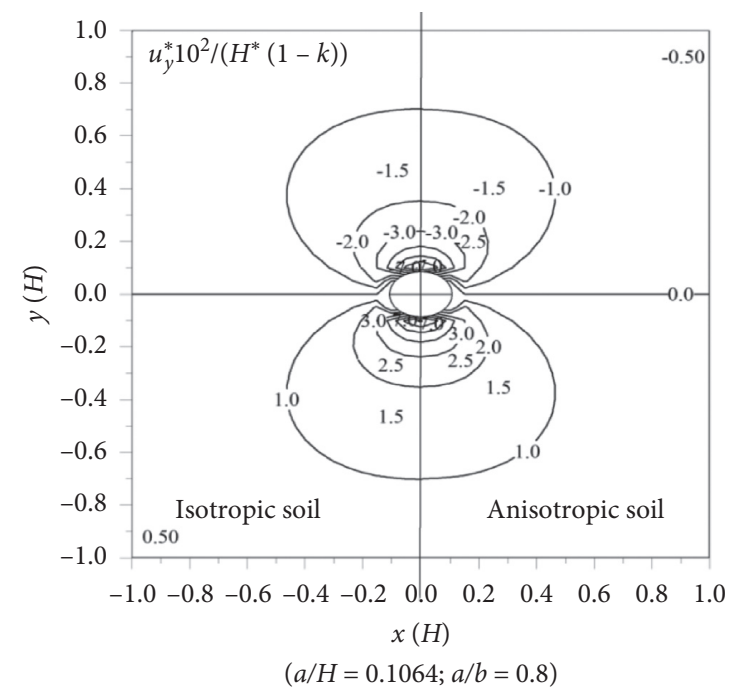

(e)

Figure 3: Analytical predictions of elastic solutions with isotropic and cross-anisotropic stiffness parameters for London clay. (a) Horizontal normal stress. (b) Vertical normal stress. (c) Shear stress. (d) Horizontal displacement. (e) Vertical displacement. 


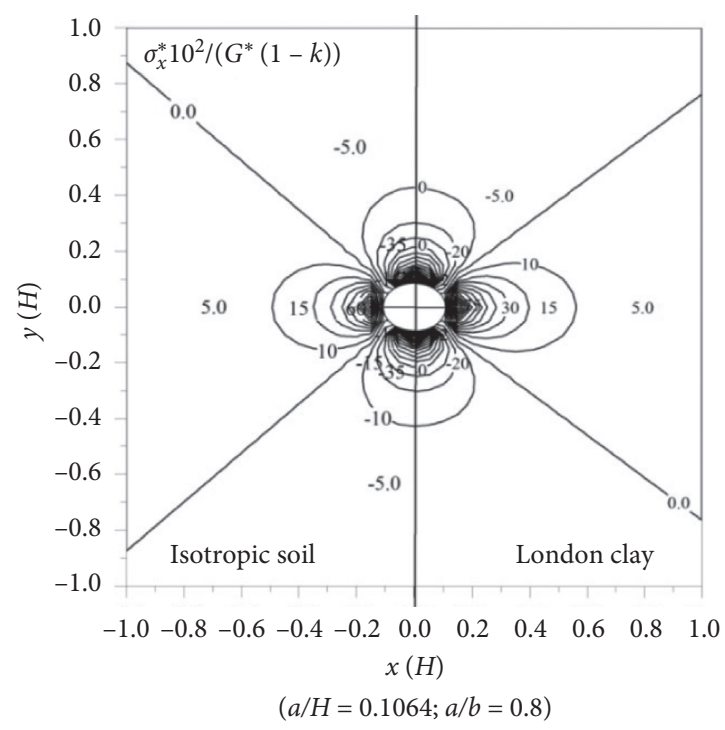

(a)

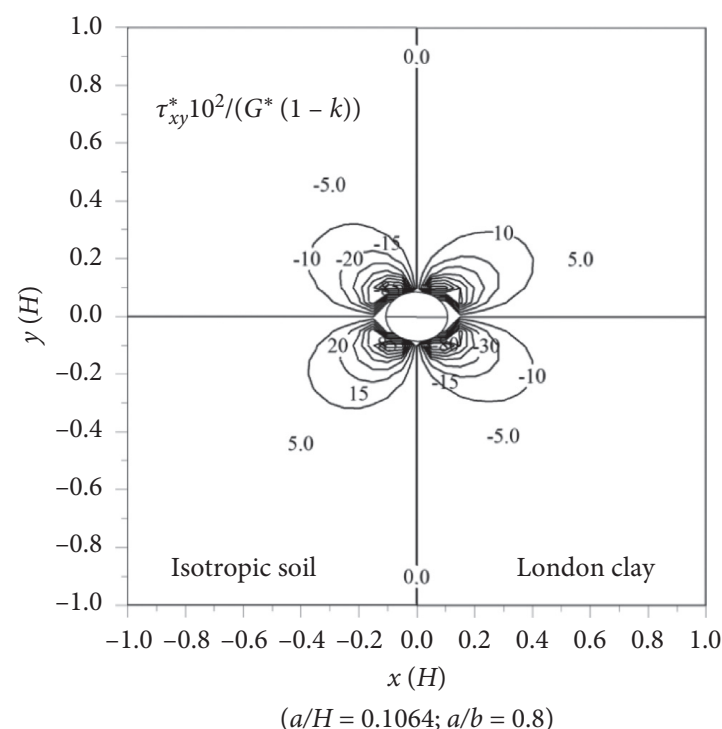

(c)

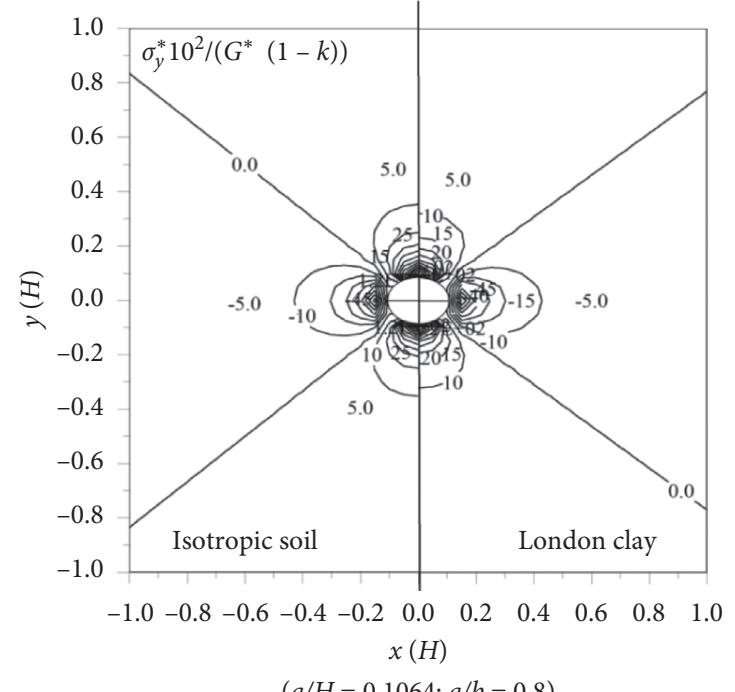

(b)

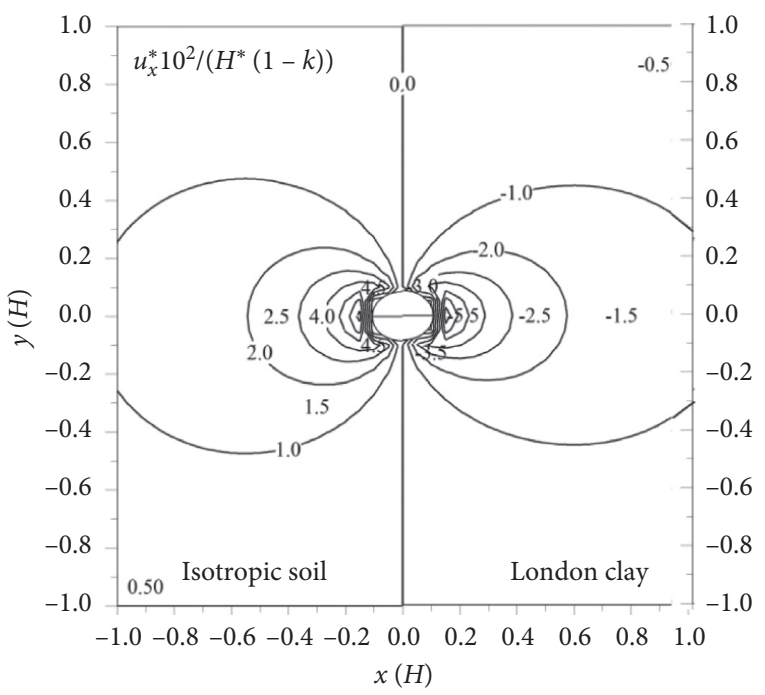

$(a / H=0.1064 ; a / b=0.8)$

(d)

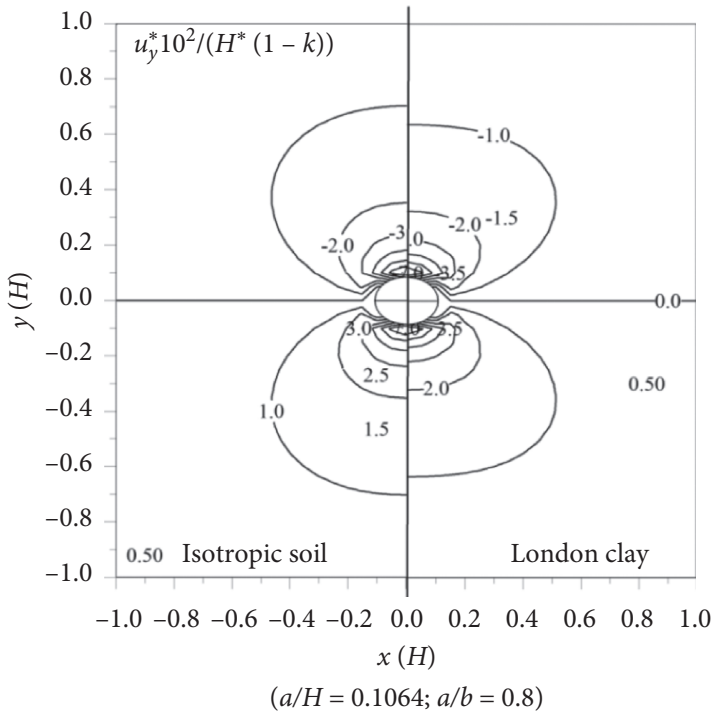

(e)

FIGURE 4: Analytical predictions of elastic solutions with isotropic and cross-anisotropic stiffness properties for London clay. (a) Horizontal normal stress. (b) Vertical normal stress. (c) Shear stress. (d) Horizontal displacement. (e) Vertical displacement. 


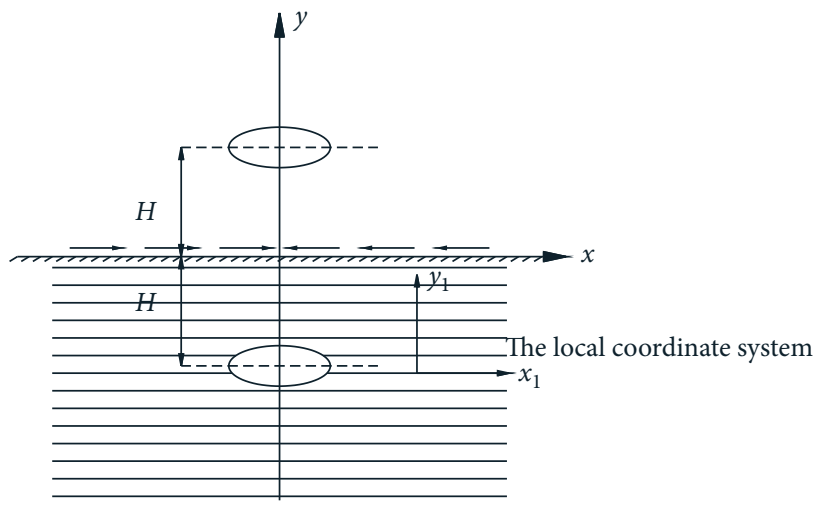

FIGURE 5: Schematic of the image method.

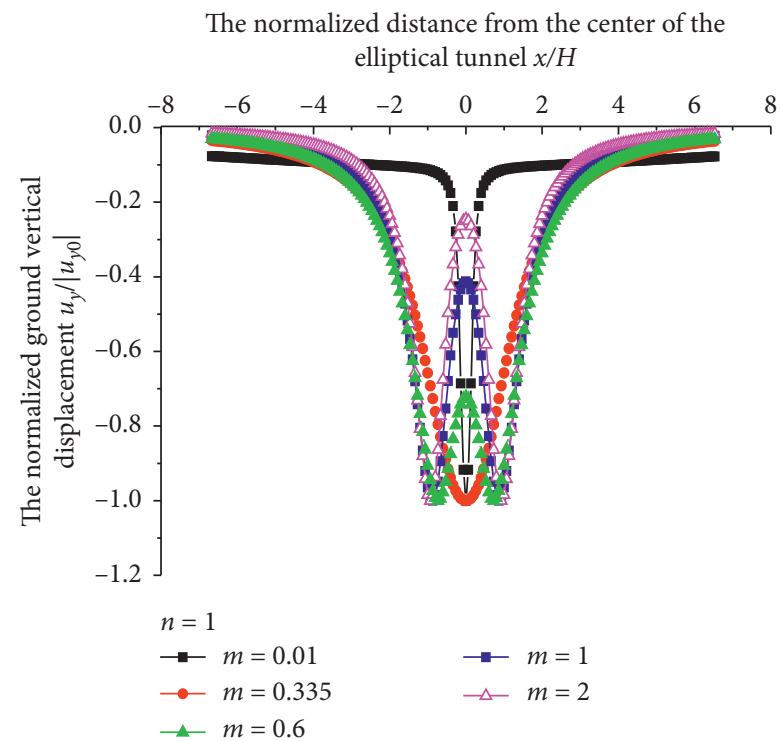

(a)

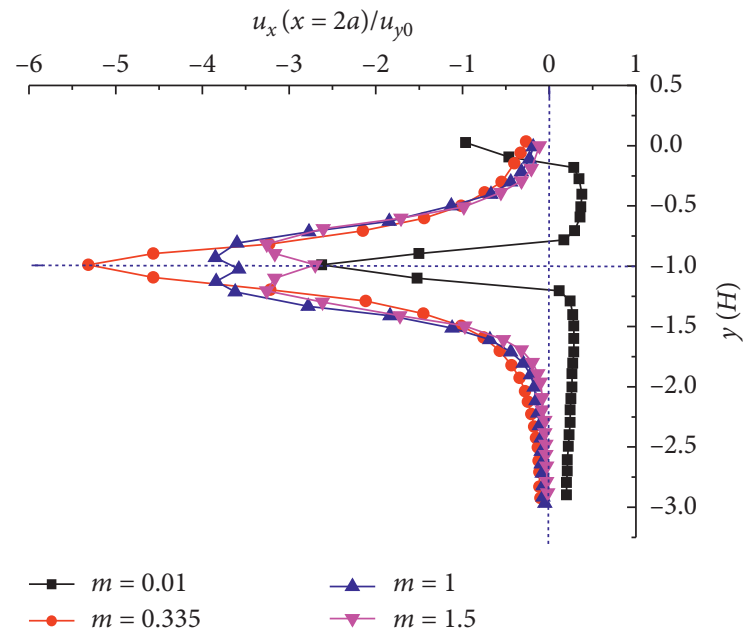

$\rightarrow m=0.335$

(b)

FiguRE 6: Influence of anisotropic stiffness ratio $(m)$ on ground settlement and distribution of underground horizontal displacement. (a) Normalized ground settlement trough. (b) Normalized lateral displacement at offset, $x / 2 R=1$.

singularity superposition technique (Figure 5 ). The displacement fields caused by a point source/sink $(0,-H)$ in a full plane and an image source $(0,+H)$ of equal and opposite deformation located equidistant above the ground surface are as follows:

$$
\begin{aligned}
u^{+}(x, y)+u^{-}(x, y) & =U(x, y+H)-U(x, y-H), \\
v^{+}(x, y)+v^{-}(x, y) & =V(x, y+H)-V(x, y-H) .
\end{aligned}
$$

The resulting normal and shear tractions along the bisecting line $y=0$ (simulating a traction-free ground surface) produced by two mirror images are as follows:

$$
\begin{aligned}
& N^{c}(x, 0)=0 \\
& T^{c}(x, 0)=-2 \tau_{x y}(x,-H) .
\end{aligned}
$$

A distribution of corrective shear tractions along the bisecting line is obtained:

$$
T_{0}^{c}(x, 0)=-T^{c}(x, 0) .
$$

The displacement results can be obtained from the following formula:

$$
\begin{aligned}
& u_{x}^{c}=2 \operatorname{Re}\left[p_{1} \Phi_{1}^{c}\left(z_{1}\right)+p_{2} \Phi_{2}^{c}\left(z_{2}\right)\right], \\
& u_{y}^{0}=2 \operatorname{Re}\left[q_{1} \Phi_{1}^{c}\left(z_{1}\right)+q_{2} \Phi_{2}^{c}\left(z_{2}\right)\right],
\end{aligned}
$$

where $\Phi_{1}^{c}\left(z_{1}\right)$ and $\Phi_{2}^{c}\left(z_{2}\right)$ are stress potential functions produced by corrective shear tractions.

The final expression for displacement field is determined by combining equations (46) and (49):

$$
\begin{aligned}
& u(x, y)=u^{+}(x, y)+u^{-}(x, y)+u^{c}(x, y), \\
& v(x, y)=v^{+}(x, y)+v^{-}(x, y)+v^{c}(x, y) .
\end{aligned}
$$

This expression is still an approximate solution as two types of tunnels have not been considered. 


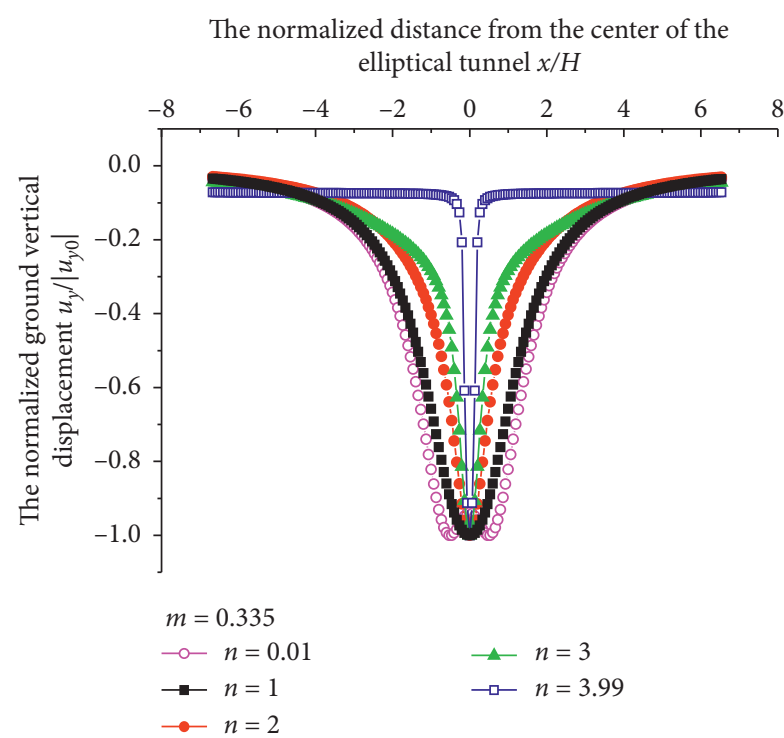

(a)

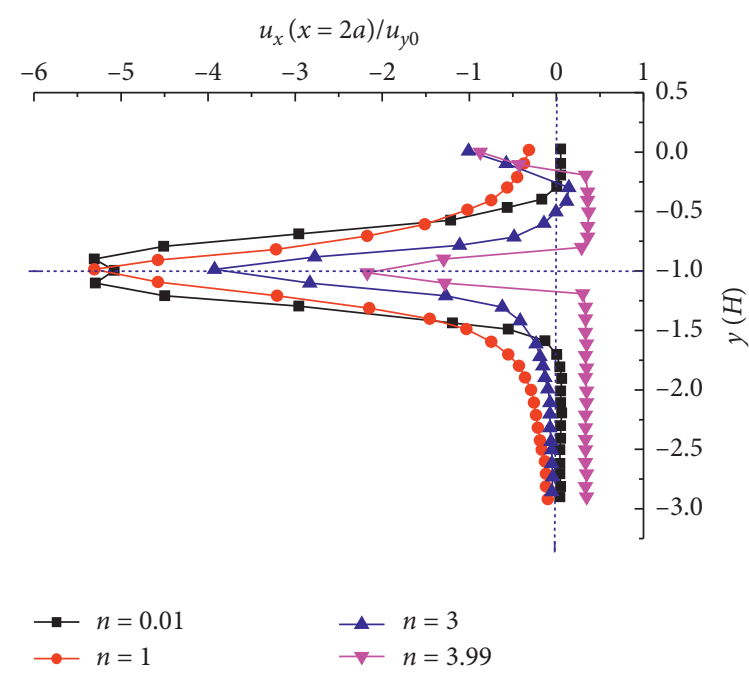

(b)

FIGURE 7: Influence of anisotropic stiffness ratio $(n)$ on ground settlement and distribution of underground horizontal displacement. (a) Normalized ground settlement trough. (b) Normalized lateral displacement at offset, $x / 2 R=1$.

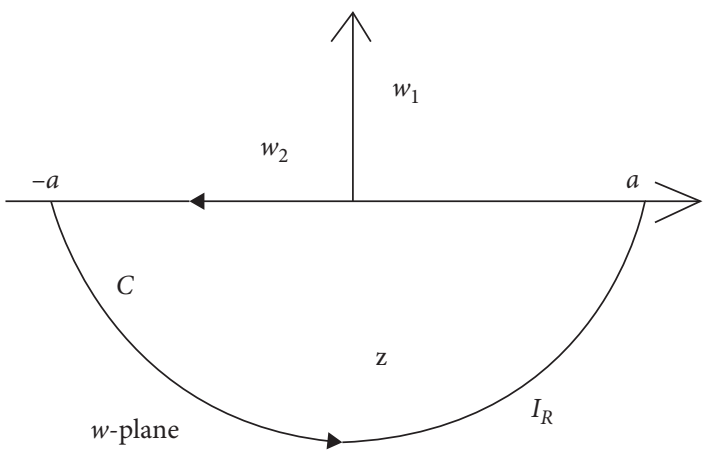

FIGURE 8: Integral path of the complex variable function.

5.2. Effect of Anisotropic Stiffness Ratios on the Predicted Displacement. We examined the effect of the anisotropic stiffness parameters, $n\left(n=E_{h} / E_{v}\right)$ and $m\left(m=G_{v h} / E_{v}\right)$, on the predictions of the ground settlement trough and subsurface horizontal displacement curves for a point offset at a distance $x=2 a$ from the center of the elliptical tunnel. These results correspond to the solutions for an elliptical tunnel with $a / H=0.1064, b / a=0.8$, and $v_{v h}=v_{h h}=0.5$.

Figures 6 and 7 show the effect of the anisotropic stiffness ratios, $m$ and $n$, on the normalized ground displacement and underground horizontal displacement at a reference vertical offset, $x=2 a$. The results indicate that the stiffness parameters, $m$ and $n$, have a significant influence on the shape of the displacement distribution. In Figure $7(\mathrm{~b}), u_{y 0}$ is the maximum vertical ground displacement calculated for varying values of the anisotropic stiffness parameter $m$, with $n=1$. The point corresponding to $y=H$ is the peak value when $m \leq 0.355$, and the solutions generate narrower troughs when $m=0.01$. The shape of the displacement trough also changes for $m>0.355$, exhibiting double peaks.
In Figure $7, u_{y 0}$ is the maximum vertical ground displacement calculated for varying values of the anisotropic stiffness parameter $n$, with $m=0.335$. It can be seen that increasing the stiffness parameter $n$ has the opposite effect compared with that for $m$. The results indicate narrow troughs when $n \geq 1$ (such as London clay), particularly for $n>3$. In contrast, for soils with $n<1$, double peaks are observed in the curves.

\section{Conclusions}

This paper has proposed an analytical method for modeling the displacement due to an unlined tunnel with an arbitrary cross section in an anisotropic plane. For this method, the following features are critical: (1) the original and deformed tunnel boundaries can be written in the form of exact or approximate polynomial mapping functions in the full plane, and the inverse mapping function must be single valued; (2) the solutions describe elastic solutions for soil under the assumption that the displacement vectors along the tunnel boundary are directed toward the center of the 
tunnel. By introducing parameters of elastic anisotropic soil, this method can easily provide elastic solutions for a tunnel with an arbitrary cross section. Several examples are used to illustrate the proposed method, and the case of an elliptical tunnel is discussed in detail to verify the accuracy of the proposed method in full plane. The anisotropic parameters of London clay have negligible influence on the horizontal stress, horizontal displacement, and shear stress; however, these parameters lead to a slightly faster attenuation of the vertical stress and vertical displacement with distance. Surface and ground displacement in half plane can be obtained using a virtual image technique. Consequently, the method developed by Zymnis [22] can be extended from a circular tunnel to a tunnel with an arbitrary cross section. Our results clearly show that the anisotropic stiffness parameters, $m$ and $n$, significantly influence the predicted displacement patterns for an unlined tunnel with a certain shape $(b / a)$ and relative embedded depth $(a / H)$.

\section{Appendix}

\section{A. Solution of the Stress Potential Function Caused by Shear Stress}

The analytical solution of $\Phi_{1}^{c}\left(z_{1}\right), \Phi_{2}^{c}\left(z_{2}\right)$ can be obtained by the following integrals [7]:

$$
\begin{aligned}
& \Phi_{1}^{c}\left(z_{1}\right)=\frac{1}{\lambda_{1}-\lambda_{2}} \frac{1}{2 \pi i} \int_{-\infty}^{\infty} \frac{\lambda_{2} f_{1}(\xi)+f_{2}(\xi)}{\xi-z_{1}} \mathrm{~d} \xi, \\
& \Phi_{2}^{c}\left(z_{2}\right)=-\frac{1}{\lambda_{1}-\lambda_{2}} \frac{1}{2 \pi i} \int_{-\infty}^{\infty} \frac{\lambda_{2} f_{1}(\xi)+f_{2}(\xi)}{\xi-z_{1}} \mathrm{~d} \xi .
\end{aligned}
$$

The normal and shear tractions are integrated along the surface of the curve and expressed as follows:

$$
\begin{aligned}
f_{1}(s) & =-\int_{\infty}^{s} N(x) \mathrm{d} x=0, \\
f_{2}(s) & =\int_{\infty}^{s} T_{0}^{c}(x) \mathrm{d} x \\
& =\int_{s}^{\infty} T^{c}(x) \mathrm{d} x .
\end{aligned}
$$

Using equation (47), we find

$$
\begin{aligned}
f_{2}(s) & =\int_{s}^{\infty} T^{c}(x) \mathrm{d} x, \\
& =-4 \operatorname{Re}\left(\int_{s}^{\infty}\left\{\lambda_{1} \Phi_{1}^{\prime}\left(z_{1}\right)+\lambda_{2} \Phi_{2}^{\prime}\left(z_{2}\right)\right\} \mathrm{d} x\right) \\
& =-4 \operatorname{Re}\left(\int_{s}^{\infty}\left\{\lambda_{1} \Phi_{1}^{\prime}\left(x,-\lambda_{1} H\right)+\lambda_{2} \Phi_{2}^{\prime}\left(x,-\lambda_{2} H\right)\right\} \mathrm{d} x\right) \\
& =-4 \operatorname{Re}\left(\lambda_{1} \Phi_{1}\left(s,-\lambda_{1} H\right)+\lambda_{2} \Phi_{2}\left(s,-\lambda_{2} H\right)\right) .
\end{aligned}
$$
obtain

Substituting the above formula into equation (A.1), we

$$
\begin{aligned}
& \Phi_{1}^{c}(z)=\frac{1}{\lambda_{1}-\lambda_{2}} \frac{1}{2 \pi \mathrm{i}} \int_{-\infty}^{\infty} \frac{f_{2}(\xi)}{\xi-z} \mathrm{~d} \xi \\
& =-\frac{1}{\pi \mathrm{i}\left(\lambda_{1}-\lambda_{2}\right)} \int_{-\infty}^{\infty} \frac{\lambda_{1} \Phi_{1}\left(\xi-\lambda_{1} H\right)+\overline{\lambda_{1} \Phi_{1}\left(\xi-\lambda_{1} H\right)}+\lambda_{2} \Phi_{2}\left(\xi-\lambda_{2} H\right)+\overline{\lambda_{2} \Phi_{2}\left(\xi-\lambda_{2} H\right)}}{\xi-z} \mathrm{~d} \xi . \\
& \Phi^{\prime}\left(w-\lambda_{k} H\right)=\frac{\Phi_{k}^{\prime}\left(\zeta_{k}\right)\left(\zeta_{k}\right)}{w_{k}^{\prime}\left(\zeta_{k}\right)} \\
& =\frac{\sum_{n=1}^{\infty}-n A_{k n}\left(\zeta_{k}\right)-(n+1)}{R \sum_{n=1}^{\infty} n\left(c_{k n} \zeta_{k}^{n-1}-d_{k n} \zeta_{k}-(n+1)\right)} \\
& =\frac{\sum_{n=1}^{\infty}-A_{k n}}{R \sum_{n=1}^{\infty}\left(c_{k n} \zeta_{k}^{2 n}-d_{k n}\right)} \text {. }
\end{aligned}
$$
respect to $w_{k}^{\prime}\left(\zeta_{k}\right)$, which yields variable function is $\mathrm{C}$, as shown in Figure 8. We also assume are two branch points $w_{k}(k=1,2)$. To obtain the branch points for the function $\phi(w)$, we differentiate $\Phi_{k}^{\prime}\left(\zeta_{k}\right)$ with 
The branch points $w_{k}(k=1,2)$ can be determined as

$$
\begin{aligned}
w_{k} & = \pm 2 \sqrt{c_{k n} d_{k n}}+\lambda_{k} H \\
& =\left\{\begin{array}{ll} 
\pm 2 \sqrt{c_{k 1} d_{k 1}}+\lambda_{k} H & n=1 \\
\pm \sqrt{\left(1+\lambda_{k}^{2}\right) a_{n} \overline{a_{n}}}+\lambda_{k} H & n \geq 2
\end{array}, \quad k=1,2 .\right.
\end{aligned}
$$

When the two branch points are located in the upper half plane and outside the integral path, $\phi(w)$ is analytic in the $w$ plane, according to the properties of the Cauchy integral:

$$
\begin{aligned}
\oint_{C} \frac{\phi(w) \mathrm{d} w}{w-z} & =2 \pi \mathrm{i} \phi(z), \\
\oint_{C} \frac{\overline{\phi(w)} \mathrm{d} w}{w-z} & =0, \\
\oint_{C} \frac{\phi(w) \mathrm{d} w}{w-z} & =\lim _{R \longrightarrow \infty}\left[\int_{R}^{-R} \frac{\phi(\xi) \mathrm{d} \xi}{\xi-z}+\int_{I_{R}} \frac{\phi(w) \mathrm{d} w}{w-z}\right] \\
& =-\int_{-\infty}^{\infty} \frac{\phi(\xi) \mathrm{d} \xi}{\xi-z} \oint_{C} \frac{\phi(w) \mathrm{d} w}{w-z}, \\
& =-\int_{-\infty}^{\infty} \frac{\phi(\xi) \mathrm{d} \xi}{\xi-z} .
\end{aligned}
$$

The final result of $\Phi_{1}^{c}\left(z_{1}\right), \Phi_{2}^{c}\left(z_{2}\right)$ can be obtained by simplifying equation (A.4) using equations (A.7) and (A.8).

\section{Data Availability}

The data used to support the findings of this study are available from the corresponding author upon request.

\section{Conflicts of Interest}

The authors declare that they have no conflicts of interest.

\section{Acknowledgments}

This work was financially supported by the National Natural Science Foundation of China (Grant no. 41702313), advanced programs from Doctoral Fund of Zhejiang Province, the R\&D Program RENSSKADE II/REMEDY at Norwegian Geotechnical Institute, and the China Postdoctoral Science Foundation (Grant nos. 2018M630468 and 2018T110407). The authors also gratefully acknowledge the support provided by Norwegian Geotechnical Institute, Research Council of Norway, and China Scholarship Council.

\section{References}

[1] A. J. Abbo, D. W. Wilson, S. W. Sloan, and A. V. Lyamin, "Undrained stability of wide rectangular tunnels," Computers and Geotechnics, vol. 53, no. 3, pp. 46-59, 2013.

[2] H. Nakamura, T. Kubota, M. Furukawa, and T. Nakao, "Unified construction of running track tunnel and crossover tunnel for subway by rectangular shape double track cross- section shield machine," Tunnelling and Underground Space Technology, vol. 18, no. 2, pp. 253-262, 2003, https://www. sciencedirect.com/science/article/abs/pii/ S0886779803000348.

[3] J. B. Si, Y. H. Zhu, C. Ji, and S. H. Zhou, "Measurement and analysis of vertical deformation of stratum induced by quasirectangular shield tunneling in soft ground," Chinese Journal of Rock Mechanics and Engineering, vol. 36, no. 6, pp. 15511559, 2017.

[4] B. Chow, "Double-O-tube shield tunneling technology in the shanghai rail transit project," Tunnelling and Underground Space Technology, vol. 21, no. 6, pp. 594-601, 2006.

[5] C. Carranza-Torres and C. Fairhurst, "The elasto-plastic response of underground excavations in rock masses that satisfy the hoek-brown failure criterion," International Journal of Rock Mechanics and Mining Sciences, vol. 36, no. 6, pp. 777-809, 1999.

[6] B.-L. Li, X.-Q. Fang, T.-F. Zhang, and S.-P. Yang, "Elastic-slip interface effect on dynamic response of underwater convey tunnel in saturated poroelastic soil subjected to plane waves," Tunnelling and Underground Space Technology, vol. 103, Article ID 103468, 2020.

[7] X. Q. Fang, T. F. Zhang, B. L. Li, and R. J. Yuan, "Elastic-slip interface effect on dynamic stress around twin tunnels in soil medium subjected to blast waves," Computers and Geotechnics, vol. 119, Article ID 103301, 2020.

[8] A. Bobet, "Lined circular tunnels in elastic transversely anisotropic rock at depth," Rock Mechanics and Rock Engineering, vol. 44, no. 2, pp. 149-167, 2011.

[9] S. G. Lekhnitskii, Theory of Elasticity of an Anisotropic Body, Wiley, Hoboken, NJ, USA, 1963.

[10] Z. Zhang and Y. Sun, "Analytical solution for a deep tunnel with arbitrary cross section in a transversely isotropic rock mass," International Journal of Rock Mechanics and Mining Sciences, vol. 48, no. 8, pp. 1359-1363, 2011.

[11] N. I. Muskhelishvili, "Some basic problems of the mathematical theory of elasticity," Mathematical Gazette, vol. 48, no. 365, p. 351, 1953.

[12] G. E. Exadaktylos and M. C. Stavropoulou, "A closed-form elastic solution for stresses and displacements around tunnels," International Journal of Rock Mechanics and Mining Sciences, vol. 39, no. 7, pp. 905-916, 2002.

[13] H. T. Manh, J. Sulem, and D. Subrin, "A closed-form solution for tunnels with arbitrary cross-section excavated in elastic anisotropic ground," Rock Mechanics \& Rock Engineering, vol. 16, no. 3, pp. 277-288, 2015.

[14] A. E. Green and G. I. Taylor, "Stress systems in aeolotropic plates. I," Proceedings of the Royal Society of London. Series A. Mathematical and Physical Sciences, vol. 173, no. 953, pp. $162-172,1939$.

[15] A. E. Green and G. I. Taylor, "Stress systems in aeolotropic plates, III," Proceedings of the Royal Society of London. Series A. Mathematical and Physical Sciences, vol. 184, no. 997, pp. 181-195, 1945.

[16] A. E. Green and G. I. Taylor, "Stress systems in aeolotropie plates. VI," Proceedings of the Royal Society of London. Series A. Mathematical and Physical Sciences, vol. 184, no. 998, pp. 289-300, 1945.

[17] C. Sagaseta, "Analysis of undraind soil deformation due to ground loss," Géotechnique, vol. 37, no. 3, pp. 301-320, 1987.

[18] A. Verruijt, "A complex variable solution for a deforming circular tunnel in an elastic half-plane," International Journal for Numerical and Analytical Methods in Geomechanics, vol. 21, no. 2, pp. 77-89, 1997. 
[19] K. H. Park, "Elastic solution for tunneling-induced ground movements in clays," International Journal of Geomechanics, vol. 4, no. 4, pp. 310-318, 2004.

[20] K.-H. Park, "Analytical solution for tunnelling-induced ground movement in clays," Tunnelling and Underground Space Technology, vol. 20, no. 3, pp. 249-261, 2005.

[21] F. Pinto and A. J. Whittle, "Ground movements due to shallow tunnels in soft ground. I: analytical solutions," Journal of Geotechnical \& Geoenvironmental Engineering, vol. 140, no. 4, Article ID 04013040, 2013.

[22] D. M. Zymnis, I. Chatzigiannelis, and A. J. Whittle, "Effect of anisotropy in ground movements caused by tunnelling," Géotechnique, vol. 63, no. 13, pp. 1083-1102, 2013.

[23] D. M. Zymnis, Evaluation of Analytical Methods to Interpret Ground Deformations Due to Soft Ground Tunneling, Cambridge University, England, UK, 2009.

[24] B. Simpson, J. H. Atkinson, and V. Jovicic, "The influence of anisotropy on calculations of ground settlements above tunnels," in Proceeding International Symposium on Geotechnical Aspects of Underground Construction in Soft Ground, R. J. Mair and R. N. Taylor, Eds., pp. 591-595, Balkema, London, UK, 1996.

[25] K. M. Lee and R. K. Rowe, "Deformations caused by surface loading and tunneling: the role of elastic anisotropy," Géotechnique, vol. 39, no. 1, pp. 125-140, 1989.

[26] D. J. Pickering, "Anisotropic elastic parameters for soil," Géotechnique, vol. 20, no. 3, pp. 271-276, 1970.

[27] R. E. Gibson, "The analytical method in soil mechanics," Ge 'otechnique, vol. 24, no. 1, pp. 115-140, 1974.

[28] A. Gasparre, S. Nishimura, N. A. Minh, M. R. Coop, and R. J. Jardine, "The stiffness of natural London clay," Géotechnique, vol. 57, no. 1, pp. 33-47, 2007.

[29] W. Zou, Q. He, M. Huang, and Q. Zheng, "Eshelby's problem of non-elliptical inclusions," Journal of the Mechanics and Physics of Solids, vol. 58, no. 3, pp. 346-372, 2010. 